\title{
Interparticle Interactions in Annealed FePt Nanoparticle Assemblies
}

\author{
Hao Zeng, Jing Li, Zhong L. Wang, J. Ping Liu, and Shouheng Sun
}

\begin{abstract}
Thermal treatment of self-assembled FePt nanoparticles reduces the interparticle distances, resulting in dramatic changes in the type and strength of interparticle interactions. Consequently, magnetic properties such as the hysteresis and magnetization reversal mechanisms are strongly affected. It is suggested that controlled annealing of self-assembled nanoparticles may offer a novel approach for producing hard magnetic nanocomposites.
\end{abstract}

Index Terms-Exchange coupling, FePt nanoparticles, magnetization reversal, self-assembly.

\section{INTRODUCTION}

C HEMICALLY ordered FePt alloys possess hard magnetic properties, with the magnetocrystalline anisotropy $K_{u}$ on the order of $5 \times 10^{7} \mathrm{erg} / \mathrm{cc}$ [1]. Recently, FePt thin films [2], [3] have drawn much attention due to their potential applications for high-density magneto-optical and magnetic recording. The high $K_{u}$ defers the emergence of the superparamagnetic limit well into terabit/in ${ }^{2}$ regime [4]. FePt alloys are also known to be permanent magnetic materials [5]. The energy products $(B H)_{\max }$ of hard-soft exchange coupled FePt multilayer films exceed 40 MGOe [6].

Chemical synthesis and self-assembly of magnetic nanoparticles may offer a novel and convenient approach for producing permanent magnetic materials. However, to reach maximum energy products for nanomaterials, the particle size and structure, and interparticle interactions have to be well controlled. It was recently reported that monodisperse FePt nanoparticles could be produced in an organic solution phase at elevated temperatures [7], [8]. The particle size and composition can be readily controlled by synthesizing parameters. Thermal annealing leads to a phase transformation from disordered face-centered cubic (fcc) to ordered face-centered tetragonal (fct) structure. Annealing also induces changes in interparticle spacing as the annealing results in the decomposition of organic coating [9]. In this paper, we study the influence of the interparticle spacing on magnetic properties. It is found that as the interparticle spacing decreases with annealing, the interactions change from magnetostatic to exchange coupling. The exchange coupled FePt nanoparticles

\footnotetext{
Manuscript received February 7, 2002; revised May 21, 2002. This work was supported in part by the U.S. Department of Defense (DoD)/Defense Advanced Research Project Agency (DARPA) under Grant DAAD19-01-1-0546.

H. Zeng and S. Sun are with the IBM T. J. Watson Research Center, Yorktown Heights, NY 10598 USA (e-mail: haozeng@us.ibm.com).

J. Li and Z. L. Wang are with the School of Materials Science and Engineering, Georgia Institute of Technology, Atlanta, GA 77005 USA.

J. P. Liu is with the Institute for Micromanufacturing, Louisiana Technical University, Ruston, LA 71272 USA.

Digital Object Identifier 10.1109/TMAG.2002.803216
}

show squarer hysteresis loop and enhanced remanence ratio, both of which are mandatory for permanent magnetic material applications.

\section{EXPERIMENTAL PROCEDURES}

The monodisperse $\mathrm{Fe}_{56} \mathrm{Pt}_{44}$ nanoparticles were synthesized by reduction of platinum acetylacetonate and decomposition of iron pentacarbonyl in the presence of oleic acid and oleyl amine stabilizers following the published procedures [7], [8]. The nanoparticles were isolated and purified by centrifugation after the addition of ethanol, and redispersed in hexane in a variety of concentrations. Deposition of this dispersion on a solid substrate and controlling solvent evaporation led to an $\mathrm{FePt}$ nanoparticle assembly. The as-deposited assemblies were annealed under a gas mixture of $5 \% \mathrm{H}_{2}$ and $95 \%$ Ar, at temperatures ranging from $500{ }^{\circ} \mathrm{C}$ to $700{ }^{\circ} \mathrm{C}$. The annealing time was fixed to be $1 \mathrm{~h}$.

The interparticle spacing, chemical ordering of a single FePt nanoparticle and coalescence of a group of FePt nanoparticles in a FePt assembly were studied by a Hitachi HF-2000 TEM at 200 kV. Quantum Design's superconducting quantum interference device (SQUID) with a field of up to $70 \mathrm{kOe}$ was used to measure hysteresis loops and remanence curves at various temperatures.

\section{RESULTS AND DISCUSSION}

Fig. 1(a)-(c) shows the TEM images of FePt assemblies of (a) as-deposited, and annealed at (b) $600{ }^{\circ} \mathrm{C}$ and (c) $700{ }^{\circ} \mathrm{C}$, respectively. The as-deposited nanoparticle assemblies have an average diameter of $4 \mathrm{~nm}$ [Fig. 1(a)]. Previous experiments have shown that annealing above $500{ }^{\circ} \mathrm{C}$ results in a phase transformation from chemically disordered fcc to ordered fct structure [7]-[9]. Further microscopic analsis indicate that annealing at higher temperatures $\left(>550^{\circ} \mathrm{C}\right)$ reduces the interparticle spacing in multilayer regions due to the decomposition of organic coating. After prolonged annealing at even higher temperatures, for example, $600{ }^{\circ} \mathrm{C}$ for $1 \mathrm{~h}$ under reducing atmosphere, particle coalescence occurs. Although annealing in this case destroys the well-defined self-assembled structure, the particles conglomerate by just touching each other. From the high resolution HR-TEM image [see Fig. 1(b)], it can be seen that the irregularly shaped particles consist of a few nanocrystallites, which are formed by several nanoparticles, with each two of them sharing common grain boundaries. No single crystal structure was observed for coalesced particles. Further increasing the temperature to $700{ }^{\circ} \mathrm{C}$ results in significant interdiffusion. Careful examination of HR-TEM of many of the 


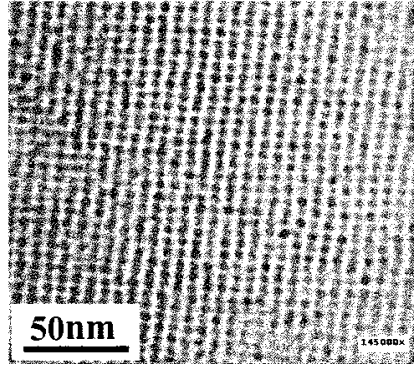

(a)

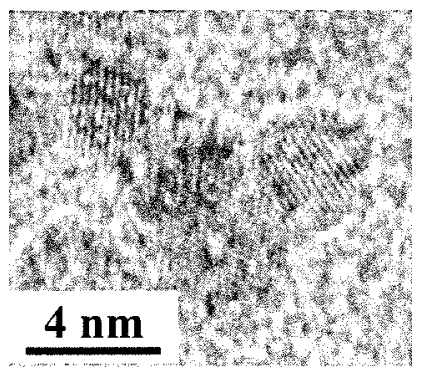

(b)

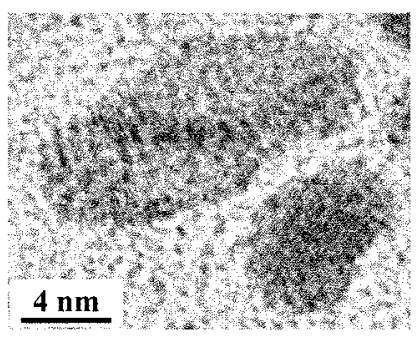

(c)

Fig. 1. (a) TEM plan-view of 4-nm FePt nanoparticle assembly. (b) HR-TEM of a conglomerated particle formed by several nanoparticles annealed at $600{ }^{\circ} \mathrm{C}$, with common grain boundaries. (c) HR-TEM of two conglomerated particles annealed at $700{ }^{\circ} \mathrm{C}$, showing single crystal structures.

big particles reveals that the grain boundaries among coalesced particles disappear, and a larger single crystal is formed, as shown in Fig. 1(c). The sizes of the coalesced particles reange from 10 to $50 \mathrm{~nm}$, with large size distributions.

The room-temperature coercivity $H_{c}$ as a function of the annealing temperature $\left(T_{a}\right)$ is shown in Fig. 2(a). The as-deposited FePt nanoparticle assembly with disordered fcc structure is superparamagnetic. Fcc to fct phase transformation occurs at the annealing temperature $T_{a}$ above $500{ }^{\circ} \mathrm{C}$, which is about $100^{\circ} \mathrm{C}$ higher than that of sputtered multilayer thin films [10]. Above $500{ }^{\circ} \mathrm{C}$, the coercivity first increases rapidly with increasing $T_{a}$, which is related to the improved ordering; then drops as $T_{a}$ further increases to above $600{ }^{\circ} \mathrm{C}$. The drop of $H_{c}$ may be a direct outcome of the particle aggregation. For a big polycrystalline particle, the exchange coupling between nanocrystallites with different crystal orientations tends to average the magnetocrystalline anisotropy and reduce the effective anisotropy of the particle assembly significantly. In fact, the anisotropy constant calculated from low temperature coercivity [11] is only $2 \times$ $10^{7} \mathrm{erg} / \mathrm{cc}$, which is several times smaller than the intrinsic $K_{u}$. The sizes of the aggregated particles can be significantly larger than the domain wall width parameter $\delta_{w}=\left(A / K_{1}\right)^{1 / 2}(1.5 \mathrm{~nm}$ if $A=10^{-6} \mathrm{erg} / \mathrm{cm}$ and $K_{1}=5 \times 10^{7} \mathrm{erg} / \mathrm{cc}$ is assumed). This means that during the magnetization reversal, domain wall can

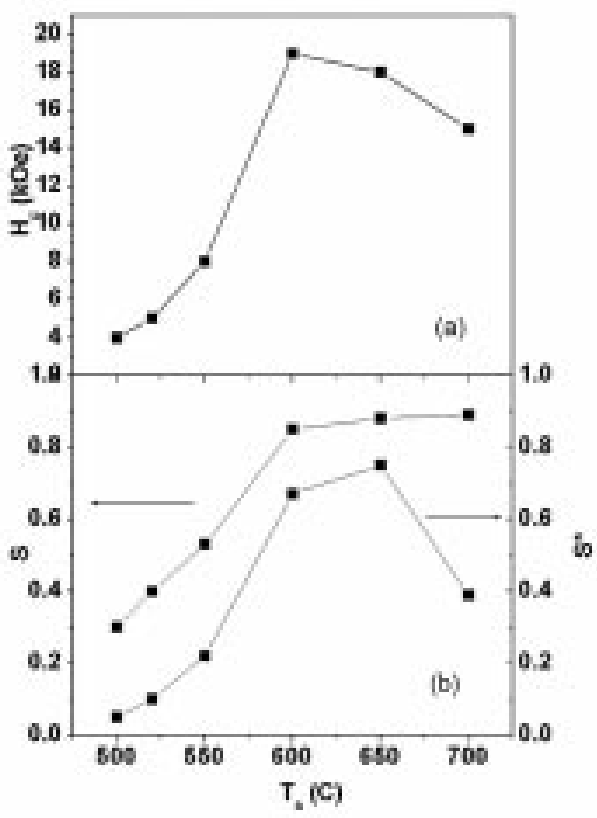

Fig. 2. (a) Room-temperature coercivity $H_{c}$ as a function of the annealing temperature $T_{a}$. (b) Remanence ratio $S$ and coercive squareness $S^{*}$ as a function of $T_{a}$.

play an important role. The initial magnetization curve for the sample annealed at $700{ }^{\circ} \mathrm{C}$ suggests that the magnetization reversal is governed by domain nucleation process [12]. Interestingly, exchange coupled thin films prepared by sputtering often show pinning reversal mechanisms [13].

Fig. 2(b) shows the room-temperature remanence ratio $S=M_{r} / M_{s}$, where $M_{r}$ is the remanent magnetization and $M_{s}$ the saturation magnetization; and the coercive squareness $S^{*}$, where $S^{*}$ is defined as $1-M_{r} /\left(H_{c}-\partial M /\left.\partial H\right|_{H c}\right)$, as a function of $T_{a} . S^{*}$ represents the switching-field-distribution (SFD): the higher $S^{*}$, the narrower SFD. For randomly oriented noninteracting particles undergoing coherent rotation, $S=0.5$ [14]. For particles exhibit magnetostatic interactions, $S$ decreases; while $S$ increases for exchange-coupled particles. As can be seen from Fig. 2(b), $S$ increases monotonically with increasing $T_{a}$, from 0.3 at $500{ }^{\circ} \mathrm{C}$ to 0.9 at $700{ }^{\circ} \mathrm{C}$, suggesting that the interactions change from predominantly magnetostatic to exchange. The SFD is finite for randomly oriented particles. The magnetostatic interactions broadens the SFD, and decreases $S^{*}$. The interparticle exchange coupling encourages cooperative switching, and narrows SFD. From Fig. 2(b), one can see that $S^{*}$ increases from 0.05 at $500{ }^{\circ} \mathrm{C}$ to 0.75 at $650{ }^{\circ} \mathrm{C}$, suggesting that the interaction changes from magnetostatic to exchange. However, $S^{*}$ decreases with further increasing $T_{a}$, likely related to the change in reversal mechanisms.

Fig. 3 shows hysteresis loops and $\delta M$ curves measured at 10 and $300 \mathrm{~K}$, for the samples annealed at $550^{\circ} \mathrm{C}$ and $600{ }^{\circ} \mathrm{C}$, respectively. $\delta M$ is defined as $M_{d}-\left(1-2 M_{r}\right)$, where $M_{d}$ is the dc demagnetization remanence and $M_{r}$ is the isothermal remanence. $\delta M$ measures qualitatively the interparticle interactions [15]. Although $H_{c}$ at room temperature for two samples differs about $50 \%, H_{c}$ values at $10 \mathrm{~K}$ are close. For $T_{a}=550^{\circ} \mathrm{C}$, the loop shape remains virtually unchanged [see Fig. 3(a)]. However, for $T_{a}=600^{\circ} \mathrm{C}$, both $S$ and $S^{*}$ drops significantly when 

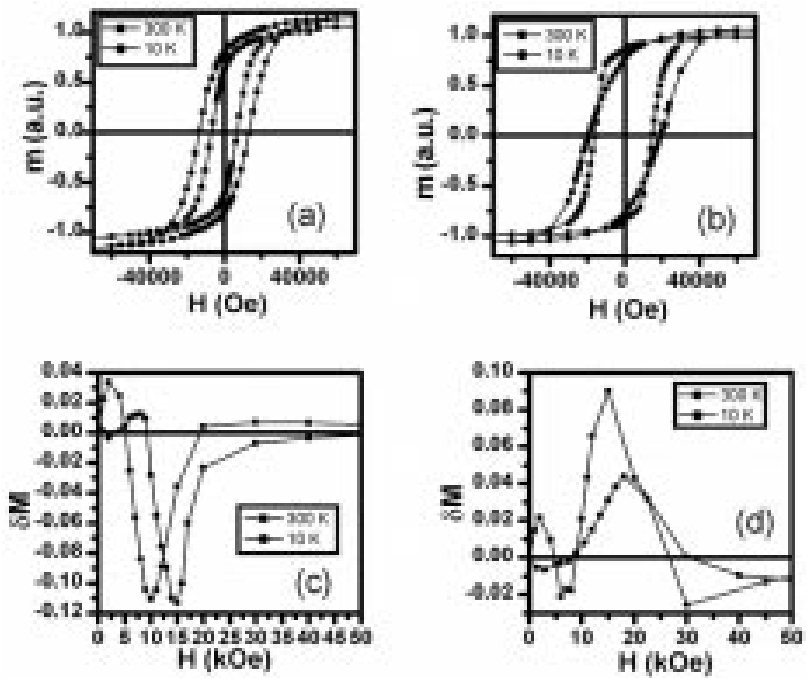

Fig. 3. (a) Hysteresis loops of a sample annealed at $T_{a}=550{ }^{\circ} \mathrm{C}$. (b) Hysteresis loops of a sample annealed at $T_{a}=600{ }^{\circ} \mathrm{C}$. (c) $\delta M$ curves for the sample annealed at $550^{\circ} \mathrm{C}$. (d) $\delta M$ curves for the sample annealed at $600^{\circ} \mathrm{C}$.

measured at $10 \mathrm{~K}$ [see Fig. 3(b)]. This rounded loop shape with a broad SFD results from nanocrystallites with a random distribution of the magnetic easy axis, which no longer couple tightly, and switch more or less independently. This is because the increase of the magnetocrystalline anisotropy at low temperatures tends to break the interparticle exchange coupling, making them switch relatively independently rather than cooperatively [16]. As can be seen from Fig. 3(c), $\delta M$ curves show negative peaks for $T_{a}=550^{\circ} \mathrm{C}$, indicating magnetostatic interactions; while for $T_{a}=600^{\circ} \mathrm{C}, \delta M$ curves show positive peaks, suggesting exchange coupling. $\delta M$ peak values for $T_{a}=550{ }^{\circ} \mathrm{C}$ remain nearly unchanged with changing measuring temperatures. This is because the magnetostatic interactions only depend on the magnetization and geometrical factors. Since the temperature dependence of $M_{s}$ is weak, while geometrical factors remain unchanged, the $\delta M$ peak values should also change very little. $\delta M$ peak values decrease significantly at low temperatures for $T_{a}=600^{\circ} \mathrm{C}$, which is consistent with the fact that the exchange coupling deteriorates with decreasing temperatures.

\section{CONCLUSION}

In conclusion, we have studied the variation of inter-particle interactions as a function of the annealing temperature for FePt nanoparticle assemblies systematically. Increasing the annealing temperature leads to the decrease of inter-particle spacing, causes nanoparticles aggregate to form polycrystalline particles, and eventually yields the formation of bigger single crystal particles. Accompanying this spacing reduction, the interactions change from magnetostatic to exchange coupling.

\section{REFERENCES}

[1] D. J. Sellmyer, C. P. Luo, and H. Zeng, "Nanoscale materials for extremely high-density recording," in Magnetic Storage Systems Beyond 2000, G. C. Hadjipanayis, Ed. Norwell, MA: Kluwer, 2001, pp. $163-170$.

[2] R. F. C. Farrow, D. Weller, R. F. Marks, M. F. Toney, A. Cebollada, and G. R. Harp, "Control of the axis of chemical ordering and magnetic anisotropy in epitaxial FePt films," J. Appl. Phys., vol. 79, pp. 5967-5969, 1996.

[3] C. P. Luo, S. H. Liou, L. Gao, Y. Liu, and D. J. Sellmyer, "Nanostructured FePt: B203 thin films with perpendicular magnetic anisotropy," Appl. Phys. Lett., vol. 77, pp. 2225-2227, 2000.

[4] D. K. Weller, A. Moser, L. Folks, M.E. Best, W. Lee, M. F. Toney, M. Schmickert, J. U. Thiele, and M. F. Doerner, "High $\mathrm{K}_{u}$ Materials Approach to 100 Gbits/in ${ }^{2}, "$ IEEE Trans. Magn., vol. 36, pp. 10-15, Jan. 2000.

[5] K. Watnanabe and M. Homma, "Perpendicular magnetization of exitaxial FePt (001) thin films with high squareness and high coercive force," Jpn. J. Phys., vol. 35, pp. L1264-L1267, 1996.

[6] P. Liu, C. P. Luo, Y. Liu, and D. J. Sellmyer, "High energy products in rapidly annealed nanoscale Fe/Pt multilayers.," Appl. Phys. Lett., vol. 72, pp. 483-485, 1998.

[7] S. Sun, C. B. Murray, D. Weller, L. Folks, and A. Moser, "Monodisperse FePt nanoparticles and ferromagnetic FePt nanocrystal superlattices," Sci., vol. 287, pp. 1989-1992, 2000.

[8] S. Sun, E. E. Fullerton, D. Weller, and C. B. Murray, "Compositionally controlled FePt nanoparticle materials," IEEE Trans. Magn., pt. 1, vol. 37, pp. 1239-1243, July 2001.

[9] Z. R. Dai, S. Sun, and Z. L. Wang, "Phase transformation coerlescence and twinning of monodisperse FePt nanocrystals," NanoLett., vol. 1, pp. 443-447, 2001

[10] C. P. Luo and D. J. Sellmyer, "Magnetic properties and structure of Fe/Pt thin films," IEEE Trans. Magn., vol. 31, pp. 2764-2766, 1995.

[11] R. W. Chantrell, D. Weller, T. J. Klemmer, S. Sun, and E.E. Fullerton, "model of the magnetic properties of FePt granular media," J. Appl. Phys., vol. 91, pp. 6866-6868, 2002.

[12] H. Zeng, T. S. Vedantam, J. P. Liu, Z. R. Dai, Z. L. Wang, and S. Sun, "Exchange-coupled FePt nanoparticle assembly," Appl. Phys. Lett., vol. 80, pp. 2583-2585, 2002, submitted for publication.

[13] J. P. Attane, Y. Samson, A. Marty, D. Halley, and C. Beigne, "Domain wall pinning on strain relaxation defects of $\mathrm{FePt}(001)-\mathrm{Pt}$ thin films," Appl. Phys. Lett., vol. 79, pp. 794-796, 2001.

[14] E. C. Stoner and E. P. Wohlfarth, "A mechanism of magnetic hysteresis in heterogeneous alloys," Phil. Trans. R. Soc. London A, Math. Phys. Sci., vol. A-240, pp. 599-642, 1948.

[15] P. E. Kelly, K. O'Grady, P. I. Mayo, and R. W. Chantrell, "Switching mechanisms in cobalt-phosphorus thin films," IEEE Trans. Magn., vol. 25, pp. 3881-3883, Sept. 1989.

[16] R. Skomski and D. J. Sellmyer, "Cooperative magnetism and Preisach model,” J. Appl. Phys., vol. 89, pp. 7263-7265, 2001. 